\title{
HOW WELL CAN RMP4 THEORY TREAT HOMOLYTIC FRAGMENTATIONS?
}

\author{
Peter M.W. GILL, Ming Wah WONG, Ross H. NOBES ${ }^{1}$ and Leo RADOM \\ Research School of Chemistry, Australian National University, Canberra, ACT 2601, Australia
}

Received 27 April 1988; in final form 18 May 1988

\begin{abstract}
The observation that fourth-order restricted Møller-Plesset perturbation theory (RMP4) gives a satisfactory description of the $\mathrm{He}_{2}^{2+}$ potential curve up to the transition structure for dissociation has led to a general examination of the applicability of RMP4 theory to the study of homolytic fragmentation in dications. It appears that, in many cases, RMP4 energies do indeed provide a useful estimate of the barrier height impeding such fragmentations and that the barrier may be estimated economically by calculating RMP4 single-point energies on the UMP2 geometries of the equilibrium and transition structures. We propose the use of a new quantity, the $\Lambda$ parameter, as an approximate measure of the rate of convergence of the RMP perturbation series and hence of the reliability of RMP4 energies.
\end{abstract}

\section{Introduction}

A convenient and commonly used procedure for incorporating the effects of electron correlation into molecular-orbital calculations in Møller-Plesset perturbation theory $[1,2]$. The usefulness of this technique depends in part on how rapidly the perturbation series (MPn) converges. In a recent article in this journal [3], we pointed out certain unsatisfactory features of the convergence behaviour of Møller-Plesset perturbation energies in describing homolytic dissociation. In particular, we found that, although the Møller-Plesset series based on an unrestricted Hartree-Fock starting point (UMP) converges smoothly, it may converge extremely slowly, leading to large errors in calculated barriers for dissociation at the practically achievable UMP4 level. On the other hand, the MP series based on a restricted Hartree-Fock starting point (RMP) was found to converge more erratically but also more rapidly so that, despite the very poor results at RHF and RMP2, a reasonably satisfactory description was obtained at the RMP4 level. We concluded that RMPn describes the homolytic dissociation of $\mathrm{He}_{2}^{2+}$ (at least up to the transition structure) better

\#1 Current address: Australian National University Supercomputer Facility, Canberra, ACT 2601, Australia. than UMP $n$, for all $n>2$. Our results were consistent with related observations of Handy and co-workers. [4].

Since RMP4 calculations are relatively costly in computational terms, it would be unfortunate if the convergence behaviour of the Moller-Plesset series with respect to geometries for the homolytic dissociation were to parallel that found for energies, since this would imply the necessity of carrying out optimizations at the RMP4 level in order to obtain satisfactory results. It would clearly be preferable if geometries could be determined at a level of theory simpler than RMP4. In particular, if HF or MP2 geometries were to prove satisfactory, we would profit not only because of their lower cost compared with RMP4 calculations but also because of the availability of analytical first derivatives at both of these levels.

We commented very briefly on some of these points in our previous paper but examine them in detail here. Transition structures for the homolytic dissociations of a selection of multiply charged cations (which are of interest to us from other points of view $[5,6])$ have been determined at a sequence of UMP and RMP levels. Such species form suitable templates for this investigation since the homolytic dissociations of multiply charged cations, unlike those of conventional neutral molecules, are generally ex- 
othermic and consequently have transition structures whose geometries and energies can be characterized. In particular, the convergence behaviour of the geometries and energies of the transition structures can be examined for both the UMP and RMP series. We find that convergence is much more rapid for UMP geometries than for UMP energies and that, for many of the cases examined, the use of UMP2 geometries for (single-point) RMP4 energy calculations does not introduce a serious error. In addition, we comment on the circumstances under which RMP4 energy calculations are likely to prove satisfactory in describing homolytic fragmentations.

\section{Method and results}

Using a modified version [7] of the GAUSSIAN 86 system of programs [8], standard ab initio calculations [9] were carried out on the three systems $\mathrm{He}_{2}^{2+}, \mathrm{CHe}_{4}^{4+}$ and $\mathrm{N}_{2} \mathrm{H}_{6}^{2+}$. The $6-31 \mathrm{G}^{* *}$ basis set was used for the dihelium dication while the $6-31 \mathrm{G}^{*}$ set was employed for the tetraheliomethane and hydrazinium systems. Additional calculations were performed on the $\mathrm{CHe}_{4}^{4+}$ tetracation and on the $\mathrm{N}_{2} \mathrm{H}_{6}^{2+}$ dication using the 6-31G and STO-3G basis sets, respectively. The geometry of the equilibrium structure of each molecule was fully optimized using each of the following levels of theory: restricted Hartree-Fock theory (RHF), second-, third- and fourth-order restricted Møller-Plesset perturbation theory (RMP2, RMP3, and RMP4, respectively) and restricted singles-and-doubles configuration interaction (RCISD). We note that the Hartree-Fock energy is equal to the first-order Møller-Plesset perturbation energy (i.e. $E(\mathrm{MP} n)$ with $n=1)$. The geometries of the transition structures for the symmetric cleavages of $\mathrm{He}_{2}^{2+}$ and $\mathrm{N}_{2} \mathrm{H}_{6}^{2+}$, and for the loss of $\mathrm{H}^{+-}$from $\mathrm{CHe}_{4}^{4+}$ were also fully optimized. These calculations may be undertaken within either the restricted (RHF) or the unrestricted (UHF) Hartree-Fock framework. We have investigated both possibilities, combining each of these with each of the five levels of theory listed above. Additionally, the barriers for dissociation were also estimated from single-point RMP4 energy calculations on the UHF and UMP $n$ geometries of the equilibrium and transition structures for each system. The results are summarized in tables 1-5. Further details of the optimized structures are presented elsewhere $[5,6]$.

In order to allow comparisons with results close to the full-CI limit (provided by CISD calculations in the case of $\mathrm{He}_{2}^{2+}$ ), geometries and barriers were calculated at the CISDTQ/6-31G level for the $\mathrm{CHe}_{4}^{4+}$ tetracation and at the CISDTQ/STO-3G level for the $\mathrm{N}_{2} \mathrm{H}_{6}^{2+}$ dication. The CISDTQ calculations (configuration interaction with all single, double, triple and quadruple excitations included) were carried out using a graphical unitary group (GUGA) CI program [10] and employing the frozen-core approximation. We believe that, for $\mathrm{CHe}_{4}^{4+}$ and $\mathrm{N}_{2} \mathrm{H}_{6}^{2+}$, CISDTQ relative energies are close to the "exact" full-CI values. This point has been examined by Harrison and Handy [11].

Finally, the RHF, RMP2, RMP3, RMP4 and fullCI energies of the $\mathrm{He}_{2}^{2+}$ dication (6-31G** basis set) and HF molecule (6-31G basis set, frozen core) were calculated at a variety of $\mathrm{He}-\mathrm{He}$ and $\mathrm{H}-\mathrm{F}$ bond lengths. The Møller-Plesset energies were found using the GAUSSIAN 86 system of programs while the

Table 1

Transition structure bond length $\left(r_{\mathrm{TS}}(\AA)\right)$, barrier height $\left(\mathrm{kJ} \mathrm{mol}^{-1}\right)$ and transition structure $\Lambda\left(1 \sigma_{\mathrm{g}}, 2 \sigma_{\mathrm{g}}\right)$ value for $\mathrm{He}_{2}^{2+} \rightarrow$ $\mathrm{He}^{+-}+\mathrm{He}^{+-}$using the $6-31 \mathrm{G}^{* *}$ basis set

\begin{tabular}{|c|c|c|c|c|c|c|}
\hline & \multicolumn{2}{|c|}{ RHF-based } & \multicolumn{2}{|c|}{ UHF-based } & \multicolumn{2}{|c|}{ RMP4//UMP a) } \\
\hline & $r_{\mathrm{TS}}$ & barrier & $r_{\mathrm{TS}}$ & barrier & barrier & $A\left(1 \sigma_{\mathrm{g}}, 2 \sigma_{\mathrm{g}}\right)$ \\
\hline $\mathrm{HF}$ & 2.186 & 794 & 0.959 & 164 & 98 & 1.73 \\
\hline MP2 & 1.362 & 337 & 1.018 & 237 & 126 & 1.60 \\
\hline MP3 & 1.196 & 199 & 1.038 & 256 & 133 & 1.56 \\
\hline MP4 & 1.131 & 147 & 1.052 & 257 & 137 & 1.53 \\
\hline CISD & 1.142 & 130 & 1.142 & 130 & & \\
\hline
\end{tabular}

a) Based on RMP4 single-point energies at UMPn geometries. 
Table 2

Transition structure bond length $\left(r_{\mathrm{Ts}}(\AA)\right)$, barrier height $\left(\mathrm{kJ} \mathrm{mol}^{-1}\right)$ and transition structure $A\left(3 \mathrm{a}_{1}, 4 \mathrm{a}_{1}\right)$ value for $\mathrm{CHe}_{4}^{4+} \rightarrow$ $\mathrm{CHe}_{3}^{3+\cdot}+\mathrm{He}^{+\cdot}$ using the $6-31 \mathrm{G}^{*}$ basis set

\begin{tabular}{|c|c|c|c|c|c|c|}
\hline & \multicolumn{2}{|c|}{ RHF-based } & \multicolumn{2}{|c|}{ UHF-based } & \multicolumn{2}{|c|}{ RMP4//UMP ${ }^{a)}$} \\
\hline & $r_{\mathrm{TS}}$ & barrier & $r_{\mathrm{TS}}$ & barrier & barrier & $A\left(3 \mathrm{a}_{1}, 4 \mathrm{a}_{1}\right)$ \\
\hline $\mathrm{HF}$ & 2.587 & 335 & 1.592 & 147 & 91 & 2.34 \\
\hline MP2 & 2.005 & 189 & 1.674 & 181 & 106 & 2.29 \\
\hline MP3 $^{\text {b) }}$ & 1.952 & 162 & 1.696 & 187 & 109 & 2.28 \\
\hline MP4 ${ }^{b)}$ & 1.793 & 113 & 1.712 & 187 & 110 & 2.26 \\
\hline CISD $^{b)}$ & 1.846 & 130 & 1.735 & 148 & & \\
\hline $\operatorname{CISD}(Q)^{b . e)}$ & 1.691 & 84 & 1.797 & 125 & & \\
\hline
\end{tabular}

a) Based on RMP4 single-point energies at UMPn geometries. ${ }^{\text {b) }}$ Frozen-core approximation used.

c) CISD with inclusion of the Davidson correction for quadruples.

Table 3

Transition structure bond length $\left(r_{\mathrm{Ts}}(\AA)\right)$, barrier height $\left(\mathrm{kJ}\right.$ mol $\left.{ }^{-1}\right)$ and transition structure $A\left(3 \mathrm{a}_{\mathrm{ig}}, 3 \mathrm{a}_{2 \mathrm{u}}\right)$ value for $\mathrm{H}_{3} \mathrm{~N}-\mathrm{NH}_{3}^{2+} \rightarrow$ $\mathrm{H}_{3} \mathrm{~N}^{+\cdot}+\mathrm{NH}_{3}^{+\cdot}$ using the $6-31 \mathrm{G}^{*}$ basis set

\begin{tabular}{|c|c|c|c|c|c|c|}
\hline & \multicolumn{2}{|c|}{ RHF-based } & \multicolumn{2}{|c|}{ UHF-based } & \multicolumn{2}{|c|}{ RMP4//UMP a) } \\
\hline & $r_{\mathrm{TS}}$ & barrier & $r_{\mathrm{Ts}}$ & barrier & barrier & $A\left(3 \mathrm{a}_{1,}, 3 \mathrm{a}_{24}\right)$ \\
\hline $\mathrm{HF}$ & 4.779 & 672 & 1.988 & 138 & 130 & 1.64 \\
\hline MP2 & 2.795 & 288 & 2.239 & 265 & 186 & 1.38 \\
\hline MP3 $^{\text {b) }}$ & 2.713 & 283 & 2.215 & 239 & 182 & 1.40 \\
\hline $\mathrm{MP}^{\mathrm{b}}{ }^{\mathbf{1}}$ & 2.531 & 209 & 2.260 & 242 & 189 & 1.36 \\
\hline CISD $^{b)}$ & 3.433 & 382 & 2.247 & 215 & & \\
\hline $\operatorname{CISD}(Q)^{b, c)}$ & 2.697 & 237 & 2.331 & 223 & & \\
\hline
\end{tabular}

a) Based on RMP4 single-point energies at UMPn geometries. b) Frozen-core approximation used.

c) CISD with inclusion of the Davidson correction for quadruples.

Table 4

Transition structure bond length $\left(r_{\mathrm{Ts}}(\AA)\right)$ and barrier height $\left(\mathrm{kJ} \mathrm{mol}^{-1}\right)$ for $\mathrm{CHe}_{4}^{4+} \rightarrow \mathrm{CHe}_{3}^{3+\cdot}+\mathrm{He}^{+\cdot}$ using the $6.31 \mathrm{G}$ basis set

\begin{tabular}{|c|c|c|c|c|}
\hline & \multicolumn{2}{|c|}{ RHF-based } & \multicolumn{2}{|c|}{ UHF-based } \\
\hline & $r_{\mathrm{TS}}$ & barrier & $r_{\mathrm{TS}}$ & barrier \\
\hline HF & 2.572 & 280 & 1.565 & 105 \\
\hline MP2 & 1.986 & 134 & 1.657 & 137 \\
\hline $\mathrm{MP3}^{\text {a) }}$ & 1.923 & 107 & 1.683 & 146 \\
\hline $\mathrm{MP}^{\mathrm{a})}$ & 1.761 & 65 & 1.699 & 147 \\
\hline CISD $^{\text {a) }}$ & 1.819 & 81 & 1.719 & 104 \\
\hline $\operatorname{CISD}(Q)^{a, b)}$ & 1.651 & 43 & 1.787 & 81 \\
\hline CISDTQ $^{a)}$ & 1.693 & 56 & & \\
\hline
\end{tabular}

a) Frozen-core approximation used.

b) CISD with inclusion of the Davidson correction for quadruples.
Table 5

Transition structure bond length $\left(r_{\mathrm{TS}}(\AA)\right)$ and barrier height $\left(\mathrm{kJ} \mathrm{mol}^{-1}\right.$ ) for $\mathrm{H}_{3} \mathrm{~N}-\mathrm{NH}_{3}^{2+} \rightarrow \mathrm{H}_{3} \mathrm{~N}^{+\cdot}+\mathrm{NH}_{3}^{+\cdot}$ using the STO-3G basis set

\begin{tabular}{llllll}
\hline & \multicolumn{2}{l}{ RHF-based } & & \multicolumn{2}{l}{ UHF-based } \\
\cline { 2 - 3 } \cline { 5 - 6 } \cline { 5 - 6 } & $r_{\text {Ts }}$ & barrier & & $r_{\text {Ts }}$ & barrier \\
\hline HF & 4.120 & 842 & & 2.051 & 174 \\
MP2 & 2.540 & 311 & & 2.211 & 266 \\
MP3 $^{\text {a) }}$ & 2.409 & 258 & & 2.220 & 269 \\
MP4 $^{\text {a) }}$ & 2.362 & 228 & & 2.241 & 268 \\
CISD $^{\text {a,b }}$ & 2.798 & 326 & & 2.306 & 245 \\
CISD(Q) $^{\text {ab) }}$ & 2.272 & 180 & & 2.374 & 250 \\
CISDTQ $^{a)}$ & 2.545 & 233 & & \\
\hline
\end{tabular}

a) Frozen-core approximation used.

b) CISD with inclusion of the Davidson correction for quadruples. 
Table 6

Total energies (RHF, RMP2, RMP3, RMP4 and full CI, in hartree) and $A\left(1 \sigma_{g}, 1 \sigma_{u}\right)$ values for $\mathrm{He}_{2}^{2+}$ as a function of the bond length (6-31G** basis set)

\begin{tabular}{|c|c|c|c|c|c|c|}
\hline$r(\dot{A})$ & RHF & RMP2 & RMP3 & RMP4 & full CI & $\Lambda\left(1 \sigma_{\mathrm{g}}, 1 \sigma_{\mathrm{u}}\right)$ \\
\hline 0.6 & -3.57851 & -3.61577 & -3.62491 & -3.62741 & -3.62840 & 3.08 \\
\hline 0.7 & -3.59475 & -3.63968 & -3.65225 & -3.65621 & -3.65806 & 2.57 \\
\hline 0.8 & -3.57055 & -3.62457 & -3.64169 & -3.64780 & -3.65103 & 2.17 \\
\hline 0.9 & -3.53096 & -3.59588 & -3.61899 & -3.62823 & -3.63338 & 1.87 \\
\hline 1.0 & -3.48792 & -3.56588 & -3.59686 & -3.61047 & -3.61773 & 1.64 \\
\hline 1.1 & -3.44705 & -3.54052 & -3.58167 & -3.60118 & -3.60961 & 1.44 \\
\hline 1.2 & -3.41079 & -3.52249 & -3.57656 & -3.60367 & -3.61020 & 1.28 \\
\hline 1.3 & $-\mathbf{3 . 3 8 0 0 9}$ & -3.51289 & -3.58301 & -3.61940 & -3.61825 & 1.14 \\
\hline 1.4 & -3.35517 & -3.51186 & -3.60138 & -3.64843 & -3.63139 & 1.01 \\
\hline 1.5 & -3.33573 & -3.51888 & -3.63111 & -3.68949 & -3.64725 & 0.91 \\
\hline
\end{tabular}

Table 7

Total energies $^{\text {a) }}$ (RHF, RMP2, RMP3, RMP4 and full CI, in hartree) and $\lambda(3 \sigma, 4 \sigma)$ values for HD as a function of the bond length (6-31G basis set)

\begin{tabular}{lllllll}
\hline$r(\dot{A})$ & RHF & RMP2 & RMP3 & RMP4 & full CI & $\lambda(3 \sigma, 4 \sigma)$ \\
\hline 0.90 & -99.98292 & -100.10968 & -100.10857 & -100.11276 & -100.11336 & 5.38 \\
1.00 & -99.97764 & -100.10967 & -100.10802 & -100.11297 & -100.11373 & 3.97 \\
1.25 & -99.92182 & -100.06665 & -100.06395 & -100.07150 & -100.07313 & 2.49 \\
1.50 & -99.85505 & -100.01321 & -100.01023 & -100.02160 & -100.02515 & 1.90 \\
1.75 & -99.79493 & -99.96782 & -99.96548 & -99.98251 & -99.98876 & 1.58 \\
2.00 & -99.74459 & -99.93415 & -99.93333 & -99.95888 & -99.96633 & 1.36 \\
2.25 & -99.70371 & -99.91229 & -99.91368 & -99.95221 & -99.95460 & 1.18 \\
2.50 & -99.67096 & -99.90120 & -99.90519 & -99.96334 & -99.94910 & 1.03 \\
2.75 & -99.64489 & -99.89949 & -99.90613 & -99.99310 & -99.94667 & 0.90 \\
\hline
\end{tabular}

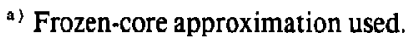

GUGA CI program was used to generate the full-CI values. The results are summarized in tables 6 and 7.

\section{Discussion}

The homolytic fissions of a hydrogen-hydrogen bond, a carbon-hydrogen bond and a carbon-carbon bond are three fundamental bond-breaking processes. Prototypical of these fragmentations are the dissociations of the hydrogen, methane and ethane molecules:

$\mathrm{H}_{2} \rightarrow \mathrm{H}^{*}+\mathrm{H}^{*}$,

$\mathrm{CH}_{4} \rightarrow \mathrm{CH}_{3}+\mathrm{H}^{\cdot}$,

$\mathrm{C}_{2} \mathrm{H}_{6} \rightarrow \mathrm{CH}_{3}+\mathrm{CH}_{3}$.
As outlined in section 1, it is preferable from a theoretical point of view to study the fragmentation processes of multiply charged ions rather than of neutral molecules because of the existence of welldefined transition structures for the former. For this reason, we have studied multiply charged isoelectronic analogues of the three reactions above, specifically:

$\mathrm{He}_{2}^{2+} \rightarrow \mathrm{He}^{+\cdot}+\mathrm{He}^{+\cdot}$,

$\mathrm{CHe}_{4}^{4+} \rightarrow \mathrm{CHe}_{3}^{3+\cdot}+\mathrm{He}^{+\cdot}$,

$\mathrm{N}_{2} \mathrm{H}_{6}^{2+} \rightarrow \mathrm{NH}_{3}^{+\cdot}+\mathrm{NH}_{3}^{+\cdot}$.

The reactions $(2 a)-(2 c)$ are formally derived from (1a)-(1c) by the replacement of $\mathrm{H}$ by $\mathrm{He}^{+}$or of $\mathrm{C}$ by $\mathrm{N}^{+}$, both of which are isoelectronic substitutions and affect the equilibrium geometries of the molecules only slightly $[5,6]$. 
In an earlier paper [3], we discussed in some detail the factors which give rise to the pattern of behaviour apparent in table 1 . For the two-electron $\mathrm{He}_{2}^{2+}$ dication, CISD is equivalent to full CI and thus yields the exact results for the basis set used. Within the RHF framework, the transition structure bond length and the barrier to dissociation both decrease rapidly from the very poor $\mathrm{RHF}$ results to the much more satisfactory RMP4 values. On the other hand, in the UHF framework, the bond length is significantly underestimated by UHF and only slowly improves along the UMP sequence. Moreover, the sequence of UMP barriers increases from a value which is already too high at the UHF level to a value at UMP4 which is almost twice the full-CI result!

The data pertaining to the dissociations of $\mathrm{CHe}_{4}^{4+}$ and $\mathrm{N}_{2} \mathrm{H}_{6}^{2+}$ (tables 2-5) indicate that the MP behaviour observed in the simple $\mathrm{He}_{2}^{2+}$ system is probably representative also of larger systems. The RHF transition structure bond length and barrier height are both reduced monotonically along the RMP sequence (although the progression is noticeably less smooth than in table 1) and the sequences of UMP transition structure bond lengths and barriers also reveal patterns similar to those in table 1. It is also interesting to compare the performance of RCISD theory with the (presumably accurate) RCISDTQ level of theory (i.e. with all triple and quadruple excitations also included in the CI calculations - a luxury which is computationally practicable only with small basis sets). While exact for the two-electron $\mathrm{He}_{2}^{2+}$ dication (table 1) and reasonable for the ten-electron $\mathrm{CHe}_{4}^{4+}$ system (tables 2 and 4), RCISD leads to gross overestimates of both the transition structure bond length and the barrier height for the eighteen-electron $\mathrm{N}_{2} \mathrm{H}_{6}^{2+}$ system (tables 3 and 5). In contrast, the RMP4 and RCISDTQ results are in quite good agreement. The large differences between RCISD and RCISDTQ highlight the well-known fact that CISD is not a size-consistent theory [9]. Apparently, the neglect of unliked quadruples in RCISD is responsible for the failure of this approach. It is interesting to note that, for $\mathrm{CHe}_{4}^{4+}$ and $\mathrm{N}_{2} \mathrm{H}_{6}^{2+}$, the popular Davidson correction [12] for unlinked quadruples (leading to RCISD (Q) results, tables 2 to 5) overcompensates and predicts transition structure bond lengths which are too short and barriers which are too low.
It is clear that, of the available Møller-Plesset model, RMP4 gives the best description of the prototypical cleavages which we are considering here. Unfortunately, of course, full RMP4 optimizations are computationally very expensive, even for rather small molecules. Nevertheless, it is apparent from tables 1-3 that both the RMP2 and UMP2 levels of theory lead to geometries which are in fair agreement with those optimized at the RMP4 level. Of these two (rather comparable) second-order treatments, we have elected to use UMP2 theory: use of RMP2 geometries would require a very rapid convergence in the RMP series from the very poor RHF starting point, which may not always be the the case. As may be seen from the second-to-last column of each of tables $1-3$, estimating the barrier by calculating RMP4 single-point energies on the UMP2 geometries of the equilibrium and transition structures provides a useful approximation to the true RMP4 barrier and removes the necessity of finding the RMP4 transition structure.

Our proposal that restricted, rather than unrestricted, Møller-Plesset perturbation theory may sometimes be the better technique for studying homolytic bond cleavages rests on the observation, in this and our earlier paper [3], that, for moderate extensions of the cleaving bond, the RMP series exhibits superior convergence behaviour to the UMP series. However, the proviso of only moderate extension is critical because, beyond a certain (molecule-dependent) critical extension, the RMP series for a homolytically cleaving molecule will always be strictly divergent. As the cleaving bond is stretched, the (occupied) molecular orbital $\psi_{\mathrm{o}}$ (which contains the pair of electrons which is being split) rises in energy while the complementary (unoccupied) orbital $\psi_{\mathrm{u}}$ falls until, when the bond length is infinite, $\psi_{\mathrm{o}}$ and $\psi_{\mathrm{u}}$ become degenerate. Because the Møller-Plesset series is an cxpansion in negative powers of the differences between orbital energies, this approach toward degeneracy of $\psi_{\mathrm{o}}$ and $\psi_{\mathrm{u}}$ will always eventually lead to divergence. Clearly then, before calculating an RMP4 single-point energy on a UHF or UMP2 structure, it is important to ascertain not only whether or not the RMP series is sufficiently rapidly convergent at that geometry but, first, whether it is convergent at all. Not to do so, i.e. to estimate the energy of a system using the first four terms of what 
might be a poorly convergent (or even divergent!) series, would be very unwise.

Although the problem of predicting the convergence properties of a general Møller-Plesset series is an unsolved one [4], we propose that the convergence behaviour of the RMP series for a singlet molecule with a stretched single bond is accurately modelled by that of the much simpler RMP series which results from using a post-Hartree-Fock window consisting of only the two orbitals $\psi_{o}$ and $\psi_{u}$. We have previously studied the electron correlation problem within such a window [3] and have found that it is possible to derive recursion formulae for the terms of the associated Møller-Plesset perturbation series.

Møller-Plesset models may be derived [9] by first introducing a generalized electronic Hamiltonin $\mathbf{H}_{\lambda}$ where

$$
\mathbf{H}_{\lambda}=\mathbf{H}_{0}+\lambda\left(\mathbf{H}-\mathbf{H}_{0}\right)
$$

and in which $\mathbf{H}$ is the exact electronic Hamiltonian and $H_{0}$ is the sum of the one-electron Fock operators. If the parameter $\lambda=0$, then $\mathbf{H}_{\lambda}=\mathbf{H}_{0}$; if $\lambda=1$, then $\mathbf{H}_{\lambda}=\mathbf{H}$. The exact energy and electronic wavefunction are then each expanded as a power series in $\lambda$ and substituted into the Schrödinger equation. Finally, $\lambda$ is set equal to unity. However, unless the radius of convergence $A$ of the energy and wavefunction expansions in $\lambda$ are substantially greater than unity, the resulting perturbation expansions will be either slowly convergent or divergent. We now derive an approximate expression for $\boldsymbol{A}$.

It is well known [13] that, for treatments based on a restricted Hartree-Fock wavefunction, the correlation energy due to single excitations is generally small compared with that from double excitations and we shall therefore neglect the singles contribution in the following treatment. In the resulting twoorbital, two-electron, doubles-only approximation, which we designate by the abbrevation " $2 \times 2$ ", the total correlation energy $E_{\text {corr }}(2 \times 2)$ is the lower eigenvalue of the correlation Hamiltonian matrix

$\mathbf{H}_{\mathrm{corr}}=\left[\begin{array}{ll}A_{11} & A_{12} \\ A_{21} & A_{22}\end{array}\right]$,

where

$A_{\text {1 } 1}=0$,
$A_{12}=A_{21}=K_{\text {ou }}$,

$A_{22}=2\left(\epsilon_{\mathrm{u}}-\epsilon_{\mathrm{o}}\right)+J_{\mathrm{oo}}+J_{\mathrm{uu}}-4 J_{\mathrm{ou}}+2 K_{\mathrm{ou}}$.

0 and $u$, as subscripts, refer to the occupied and unoccupied molecular orbitals, respectively. $J_{i j}$ and $K_{i j}$ represent the usual Coulomb and exchange integrals and $\epsilon_{i}$ is the energy of the orbital $\psi_{i}$. Letting

$\alpha=\frac{1}{2}\left(J_{\mathrm{oo}}+J_{\mathrm{uu}}\right)-2 J_{\mathrm{ou}}+K_{\mathrm{ou}}$,

$x=\left(\epsilon_{\mathrm{o}}-\epsilon_{\mathrm{u}}\right)^{-1}$,

$K=K_{\text {ou }}$,

gives

$\mathbf{H}_{\text {corr }}=\left[\begin{array}{cc}0 & K \\ K & 2 \alpha-2 / x\end{array}\right]$.

Thus,

$E_{\text {corr }}^{2}+(2 / x-2 \alpha) E_{\text {corr }}-K^{2}=0$.

Eq. (8) is a quadratic equation in $E_{\text {corr }}$ The restricted Møller-Plesset perturbation series $\operatorname{RMP}(2 \times 2)$ is just the Taylor series expansion of $E_{\text {corr }}$ in powers of $x$. The series will be convergent if, and only if, $|x|$ is less than some radius of convergence $x_{\text {crit }}$. The discriminant of the quadratic equation (8)

$D(x)=(2 / x-2 \alpha)^{2}+4 K^{2}$

has roots

$x_{ \pm}=(\alpha \pm \mathrm{i} K)^{-1}$,

whence

$x_{\text {crit }}=\left|x_{ \pm}\right|=\left(\alpha^{2}+K^{2}\right)^{-1 / 2}$.

Consequently, the RMP $(2 \times 2)$ series will converge if, and only if,

$|x|<\left(\alpha^{2}+K^{2}\right)^{-1 / 2}$.

After dividing both sides of (12) by $|x|$, it follows that the radius of convergence of the $\operatorname{RMP}(2 \times 2)$ parameter $\lambda$ is given by

$\Lambda\left(\psi_{\mathrm{o}}, \psi_{\mathrm{u}}\right)=\left(\epsilon_{\mathrm{u}}-\epsilon_{\mathrm{o}}\right)\left(\alpha^{2}+K^{2}\right)^{-1 / 2}$.

The $A$ parameter provides a quantitative measure of the RMP $(2 \times 2)$ convergence behaviour:

(i) If $A$ is much greater than unity, the $\operatorname{RMP}(2 \times 2)$ series will converge rapidly. 
(ii) If $\Lambda$ is a little greater than unity, the $\operatorname{RMP}(2 \times 2)$ series will converge slowly.

(iii) If $A$ is less than unity, the $\operatorname{RMP}(2 \times 2)$ series will diverge.

In order to calculate $A$ using (13), the six quantities on the right-hand side of (5c) are required. These are all straightforward to compute and we have added a small section of code to the population analysis link of the GAUSSIAN 86 package to find $A$ automatically at the conclusion of RHF calculations.

In the final column of tables $1-3$, we give the $\Lambda$ values calculated at the UMPn geometries of the transition structures for fragmentation of $\mathrm{He}_{2}^{2+}$, $\mathrm{CHe}_{4}^{4+}$ and $\mathrm{N}_{2} \mathrm{H}_{6}^{2+}$. In all cases, the ortibals $\psi_{0}$ and $\psi_{\mathrm{u}}$ correspond to the $\sigma$ and $\sigma^{*}$ molecular orbitals of the breaking bond. That all of the $A$ values in tables 1-3 are greater than unity indicates that the calculated RMP4 barriers derive from convergent RMP series, but it is difficult to decide at this stage, simply from the numerical values of $A$, whether or not the series are sufficiently rapidly convergent that fourthorder treatments are likely to give satisfactory results.

To investigate the question further, we have studied the quantitative relationships of $A$, both with respect to the length of the breaking bond and with respect to the performance of RMP $n$ theory, for two diatomic molecules ( $\mathrm{He}_{2}^{2+}$ and $\mathrm{HF}$ ) for which fullCI calculations are computationally feasible. The total energies calculated as a function of bond length in the $\mathrm{He}_{2}^{2+}$ and HF molecules, at the RHF, RMP2, RMP3, RMP4 and full-CI levels, are given in tables 6 and 7 . Additionally, the values of the $A$ parameter corresponding to each bond length are shown in the rightmost column of each table. These two quite different diatomic systems were examined in order to assess the generality of the RMPn convergence behaviour.

As the bond length in cach system is increased, the $A$ parameter falls monotonically (tables 6 and 7 ) from a satisfactory value in the vicinity of the equilibrium structure $\left(A \approx 2.6\right.$ for $\mathrm{He}_{2}^{2+}$ and $\approx 5.2$ for $\mathrm{HF}$ ) to a value less than unity when the bond is sufficiently stretched ( $r>1.4 \AA$ for $\mathrm{He}_{2}^{2+}$ and $>2.5 \AA$ for HF). Beyond these critical bond lengths, our model predicts that the restricted Møller-Plesset series for the two systems are divergent. In figs. 1 and 2 , we plot the unrecovered electron correlation energy $\left(E_{\mathrm{RMP} n}-\mathrm{E}_{\mathrm{CI}}\right)$ against the value of the associated $A$ parameter for each of $\mathrm{He}_{2}^{2+}$ and HF, for $n=1$ to 4 . It is clear from these graphs that, for example, for the two systems examined, the RMP2 and RMP3

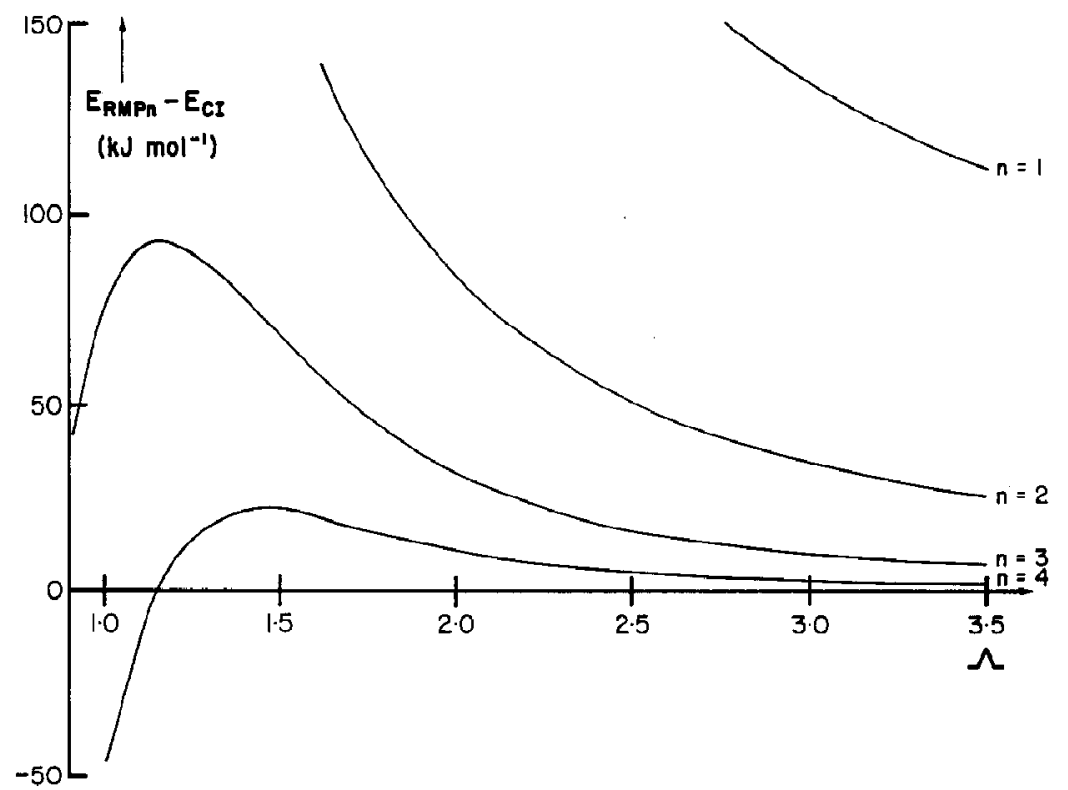

Fig. 1. Unrecovered electron correlation energy in RMPn calculations as a function of the $\Lambda\left(1 \sigma_{g}, 1 \sigma_{u}\right)$ parameter for the $H e_{2}^{2+}$ dication using the $6-31 \mathrm{G}^{* *}$ basis set. 


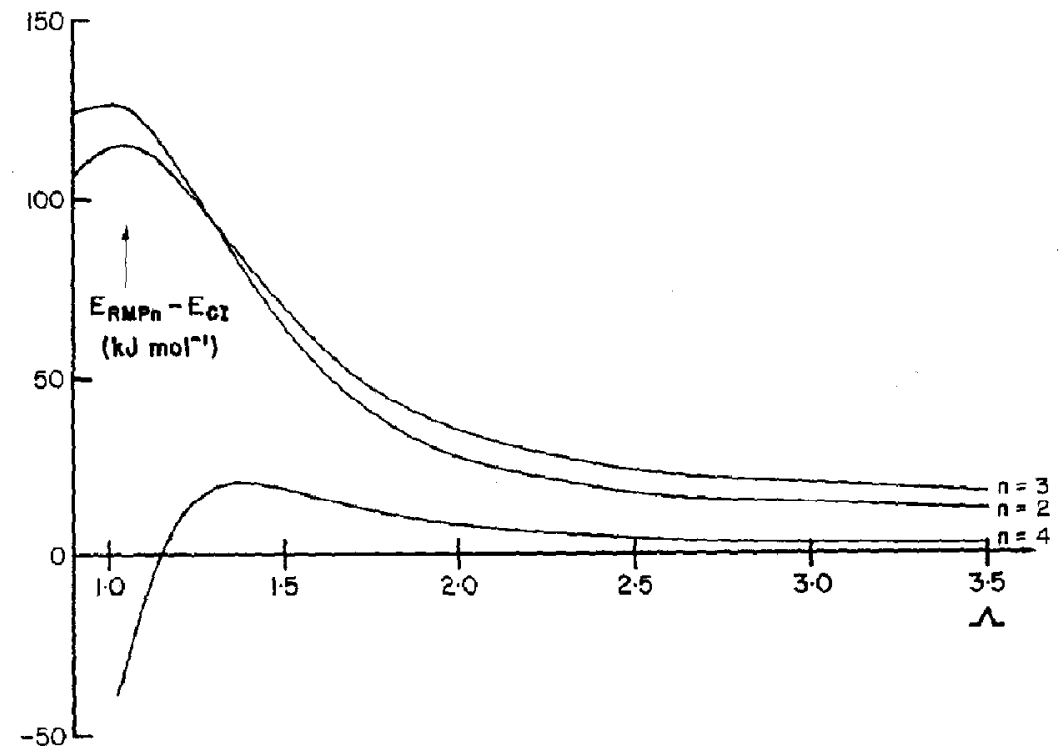

Fig. 2. Unrecovered valence-electron correlation energy in RMPn calculations as a function of the $A(3 \sigma, 4 \sigma)$ parameter for the HF molecule using the $6-31 \mathrm{G}$ basis set.

energies lie above the exact energies (i.e. $E_{\mathrm{RMP}_{n}}$ $-E_{\mathrm{Cl}}>0$ ) for all $A>1$ but that RMP4 energies lie below the full-CI limit when $A$ is less than $\approx 1.15$.

If we are interested in obtaining reliable relative energies of structures corresponding to different geometries, then it is obviously important that the unrecovered correlation energies at the various geometries be as similar as possible. This is equivalent to requiring that the graphs in figs. 1 and 2 be as uniformly flat as possible and, clearly, this is achieved much more satisfactorily at RMP4 than at RMP2 or RMP3. For example, in a previous paper [3], we found that RMP2 and RMP3 seriously overestimate the dissociation barricr of $\mathrm{He}_{2}^{2+}$ but that RMP4 is much more satisfactory (table 1). This arises because RMP2 and RMP 3 recover much less correlation energy when $A \approx 1.5$ (the value near the transition structure) than when $A \approx 2.5$ (the value near the equilibrium structure) - in fact, 200 and 70 $\mathrm{kJ} \mathrm{mol}^{-1}$ less, respectively. On the other hand, the flatness of the $n=4$ curve in fig. 1 results in the RMP4 error over the same range being only $17 \mathrm{~kJ} \mathrm{~mol}^{-1}$.

We conclude, on the basis of the results for the $\mathrm{He}_{2}^{2+}$ and HF molecules, that RMP4 theory is likely to offer a reliable treatment of those regions of potential surfaces where the $A$ parameter is greater than $\approx 1.2-1.3$. However, use of RMP4 theory at geometries where the $A$ parameter is less than 1.1 is not recommended.

\section{Conclusions}

In this paper, we have explored the possibility of using restricted Moller-Plesset ( $\mathrm{RMP}$ ) perturbation theory to study the homolytic fragmentations of dications. Although the RMP series for a homolytically cleaving molecule is found to diverge for structures in which the bond breaking is sufficiently advanced, it may often converge satisfactorily in the vicinity of the transition structure. We have introduced a simple "back-of-the-envelope" quantitative measure, the $A$ parameter, of the rate of convergence of an RMP series, as a guide for deciding whether or not RMP4 energies are likely to be reliable.

\section{Acknowledgement}

We thank Professor John Pople and Dr. Michael Collins for helpful discussions. We gratefully acknowledge a generous allocation of time on the 
Fujitsu VP-100 of the Australian National University Supercomputer Facility.

\section{References}

[1] C. Møller and M.S. Plesset, Phys. Rev. 46 (1934) 618.

[2] J.A. Pople, J.S. Binkley and R. Seeger, Intern. J. Quantum Chem. Symp. 10 (1976) 1;

J.A. Pople, R. Krishnan, H.B. Schlegel and J.S. Binkley, Intern. J. Quantum Chem. 14 (1978) 545;

R. Krishnan, M.J. Frisch and J.A. Pople, J. Chem. Phys. 72 (1980) 4244.

[3] P.M.W. Gill and L. Radom, Chem. Phys. Letters 132 (1986) 16.

[4] P.L. Knowles, K. Somasundram, N.C. Handy and K. Hirao, Chem. Phys. Letters 113 (1985) 8;

N.C. Handy, P.J. Knowles and K. Somasundram, Theoret. Chim. Acta 68 (1985) 87

[5] M.W. Wong, R.H. Nobes and L. Radom, J. Chem. Soc. Chem. Commun. (1987) 233.
[6] P.M.W. Gill and L. Radom, J. Am. Chem. Soc., submitted for publication.

[7] R.H. Nobes, N.V. Riggs, B.J. Smith and M.W. Wong, unpublished; J. Baker, J. Comput. Chem. 7 (1986) 385.

[8] M.J. Frisch, J.S. Binkley, H.B. Schlegel, K. Raghavachari, C.F. Melius, R.L. Martin J.J.P. Stewart, F.W. Bobrowicz, C.M. Rohlfing, L.R. Kahn, D.J. Defrees, R. Seeger, R.A. Whiteside, D.J. Fox. E.M. Fleuder and J.A. Pople, GAUSSIAN 86 (Carnegie-Mellon Quantum Chemistry Publishing Unit, Pittsburgh, PA, 1984).

[9] W.J. Hehre, L, Radom, P. von R. Schleyer and J.A. Pople, Ab initio molecular orbital theory (Wiley, New York, 1986).

[10] P. Saxe, D.J. Fox, H.F. Schaefer III and N.C. Handy, J. Chem. Phys. 77 (1982) 5584.

[11] R.J. Harrison and N.C. Handy, Chem. Phys. Letters 95 (1983) 386.

[12] S.R. Langhoff and E.R. Davidson, Intern. J. Quantum Chem. 8 (1974) 61.

[13] A. Szabo and N.S. Ostlund, Modern quantum chemistry: introduction to advanced electronic structure theory (Macmillan, New York, 1982). 